\title{
OPEN Author Correction: Non-alcoholic fatty liver disease and cerebral small vessel disease in Korean cognitively normal individuals
}

\author{
Hyemin Jang $\mathbb{1}^{1,2}$, Danbee Kang ${ }^{3}$, Yoosoo Chang $\mathbb{1}^{5}$, Yeshin Kim ${ }^{1,2}$, Jin San Lee ${ }^{6}$, \\ Ko Woon Kim ${ }^{7}$, Young Kyoung Jang ${ }^{1,2}$, Hee Jin Kim ${ }^{1,2}$, Duk L. Na ${ }^{1,2}$, Hee Young Shin ${ }^{4}$, \\ Mira Kang ${ }^{4}$, Eliseo Guallar $3,9,10,11,12$, Juhee Cho ${ }^{3,8,9}$ \& Sang Won Seo ${ }^{1,2}$
}

Correction to: Scientific Reports https://doi.org/10.1038/s41598-018-38357-x, published online 12 February 2019

The Acknowledgements section of this Article is incomplete.

"This work was supported by the National Research Foundation of Korea (NRF) grant funded by the Korea government (MSIP) (NRF-2017R1A2B2005081) and the Brain Research Program through the National Research Foundation of Korea (NRF) funded by the Ministry of Science, ICT \& Future Planning (2016M3C7A1913844)."

should read:

"This work was supported by the National Research Foundation of Korea (NRF) grant funded by the Korea government (MSIP) (NRF-2017R1A2B2005081), the Brain Research Program through the National Research Foundation of Korea (NRF) funded by the Ministry of Science, ICT \& Future Planning (2016M3C7A1913844), and a fund (2018-ER6203-00) by Research of Korea Centers for Disease Control and Prevention.”

(i) Open Access This article is licensed under a Creative Commons Attribution 4.0 International License, which permits use, sharing, adaptation, distribution and reproduction in any medium or format, as long as you give appropriate credit to the original author(s) and the source, provide a link to the Creative Commons license, and indicate if changes were made. The images or other third party material in this article are included in the article's Creative Commons license, unless indicated otherwise in a credit line to the material. If material is not included in the article's Creative Commons license and your intended use is not permitted by statutory regulation or exceeds the permitted use, you will need to obtain permission directly from the copyright holder. To view a copy of this license, visit http://creativecommons.org/licenses/by/4.0/.

(c) The Author(s) 2019

\footnotetext{
${ }^{1}$ Department of Neurology, Samsung Medical Center, Sungkyunkwan University School of Medicine, Seoul, 06351, Korea. ${ }^{2}$ Neuroscience Center, Samsung Medical Center, 06351, Seoul, Korea. ${ }^{3}$ Center for Clinical Epidemiology, Samsung Medical Center, 06351, Seoul, Korea. 'Health Promotion Center, Samsung Medical Center, 06351, Seoul, Korea. ${ }^{5}$ Department of Occupational and Environmental Medicine, Kangbuk Samsung Hospital, Sungkyunkwan University, School of Medicine, Seoul, Korea. ${ }^{6}$ Department of Neurology, Kyung Hee University Hospital, Seoul, Korea. ${ }^{7}$ Department of Neurology, Chonbuk National University Hospital, Chonbuk National University Medical School, Jeonju, Korea. ${ }^{8}$ Department of Health Sciences and Technology, Sungkyunkwan University, Seoul, 06351, Korea. ${ }^{9} \mathrm{Clinical}$ Research Design and Evaluation, SAIHST, Sungkyunkwan University, Seoul, 06351, Korea. ${ }^{10}$ Department of Epidemiology, Johns Hopkins Medical Institutions, Baltimore, USA. ${ }^{11}$ Department of Medicine, Johns Hopkins Medical Institutions, Baltimore, USA. ${ }^{12}$ Welch Center for Prevention, Epidemiology and Clinical Research, Johns Hopkins Medical Institutions, Baltimore, USA. Hyemin Jang, Danbee Kang, Juhee Cho and Sang Won Seo contributed equally. Correspondence and requests for materials should be addressed to J.C. (email: alfadur2j@ gmail.com) or S.W.S. (email: sangwonseo@empal.com)
} 